PROCEEDINGS OF THE

AMERICAN MATHEMATICAL SOCIETY

Volume 140, Number 9, September 2012, Pages 3219-3227

S 0002-9939(2012)11152-9

Article electronically published on January 13, 2012

\title{
FINITE SUMS OF PROJECTIONS IN PURELY INFINITE SIMPLE C*-ALGEBRAS WITH TORSION $K_{0}$
}

\author{
VICTOR KAFTAL, P. W. NG, AND SHUANG ZHANG
}

(Communicated by Marius Junge)

\begin{abstract}
Assume that $\mathcal{A}$ is a purely infinite simple $\mathrm{C}^{*}$-algebra whose $K_{0}$ is a torsion group, namely, contains no free element. Then a positive element $a \in \mathcal{A}$ can be written as a finite sum of projections in $\mathcal{A}$ if and only if either $a$ is a projection or $\|a\|>1$.
\end{abstract}

\section{INTRODUCTION}

In [5, Theorem 1.1] Fillmore proved that a positive finite-rank operator $a \in$ $B(H)$ can be expressed as finite sums of projections if and only if $\operatorname{Tr}(a) \in \mathbb{N}$ and $\operatorname{Tr}(a) \geq \operatorname{rank}(a)$, where $\operatorname{Tr}$ is the standard integer-valued trace.

An immediate natural question is: which infinite-rank operators can also be expressed as finite sums or infinite sums of projections, where the infinite sums of projections are assumed to converge in the strong topology? Motivated by needs in frame theory, this question was posed by Dykema, Freeman, Kornelson, Larson, Ordower, and Weber in 4, where they also proved a sufficient condition.

Recently, we obtained in [6, Theorem 1.1] the complete characterization: a positive operator $a \in B(H)$ is a (possibly infinite) sum of projections if and only if

$$
\operatorname{Tr}\left(a_{+}\right) \geq \operatorname{Tr}\left(a_{-}\right) \quad \text { and if } \operatorname{Tr}\left(a_{+}\right)<\infty \text { then } \operatorname{Tr}\left(a_{+}\right)-\operatorname{Tr}\left(a_{-}\right) \in \mathbb{N} \cup\{0\} .
$$

Here $a_{+}:=(a-I) \chi_{(1, \infty)}(a)$, and $a_{-}:=(I-a) \chi_{[0,1)}(a)$ where $\chi(a)$ is the spectral measure of $a$.

A similar characterization, but without the integrality condition, can be given for all positive diagonalizable operators in $\sigma$-finite von Neumann factors. In particular, in type III factors a positive operator $a$ can be expressed as a sum of projections if and only if either $a$ is a projection or $\|a\|>1$.

Turning to another direction, we generalized the $B(H)$ results to bounded module maps on Hilbert $C^{*}$-modules. In [7] we considered the multiplier algebras of purely infinite and simple $\mathrm{C}^{*}$-algebras, equipped with the strict topology (in analogy to the strong operator topology on $B(H)$ ) and obtained the following complete characterization ([7, Theorem 1.1 ]): if $\mathcal{A}$ is a $\sigma$-unital, nonunital, purely infinite simple $\mathrm{C}^{*}$-algebra and $a$ is a positive element of $\mathcal{M}(\mathcal{A})$, then $a$ is a sum of projections belonging to $\mathcal{A}$, with convergence in the strict topology, if and only if one of

Received by the editors December 9, 2010 and, in revised form, March 28, 2011.

2010 Mathematics Subject Classification. Primary 46L05; Secondary 47C15.

The third author was supported by a Taft Center Travel Grant when the article was presented in Beijing, China, in the summer of 2010.

(C)2012 American Mathematical Society Reverts to public domain 28 years from publication 
the following mutually exclusive conditions holds:

(i) $\|a\|_{e}>1$.

(ii) $\|a\|_{e}=1$ and $\|a\|>1$.

(iii) $a \in \mathcal{M}(\mathcal{A}) \backslash \mathcal{A}$ is a projection.

(iv) $a$ is the sum of finitely many projections belonging to $\mathcal{A}$.

Here $\|\cdot\|_{e}$ denotes the essential norm on $\mathcal{M}(\mathcal{A})$, namely $\|a\|_{e}=\|\pi(a)\|$, where $\pi: \mathcal{M}(\mathcal{A}) \rightarrow \mathcal{M}(\mathcal{A}) / \mathcal{A}$ is the canonical quotient map.

A harder problem is to characterize the case (iv) above, that is, which positive elements in a $\mathrm{C}^{*}$-algebra $\mathcal{A}$ are finite sums of projections in $\mathcal{A}$. This question is still open even in $B(H)$.

A sufficient condition for $a \in B(H)^{+}$to be a finite sum of projections is that $\|a\|_{e}>1$. This result was reported in a survey article [13. Theorem 4.12] by Wu quoting unpublished joint work of Choi and Wu in 1988. The proof of this sufficient condition was presented in their recent paper [1, Theorem 2.2].

The special but important case, $\alpha I$ with $\alpha>1$, follows also from a delicate analysis in [10, [11, and several other papers which characterized the set

$$
\Sigma_{n}:=\{\alpha \in \mathbb{R} \mid \alpha I \text { is the sum of } n \text { projections }\} .
$$

A consequence of their analysis yields that $\bigcup_{1}^{\infty} \Sigma_{n}=[1, \infty)$; i.e., $\alpha I$ is a finite sum of projections if and only if $\alpha \geq 1$.

An independent and different proof of the sufficient condition by Choi and Wu was given by the authors of the present paper in [8]: if $M$ is an infinite $\sigma$-finite von Neumann factor and $a \in M$ is positive, then a sufficient condition for $a$ to be a finite sum of projections in $M$ is, respectively,

(i) $\|a\|_{e}>1$ when $M$ is of type $\mathrm{I}_{\infty}$ (where $\|\cdot\|_{e}$ denotes the usual essential norm of $B(H))$;

(ii) $\|a\|_{e}>1$ when $M$ is of type $\mathrm{II}_{\infty}$ (where $\|\cdot\|_{e}$ denotes the essential norm relative to the closed ideal $J(M)$ generated by all finite projections of $M$ );

(iii) $\|a\|>1$ when $M$ is of type III. Moreover, $a \in M^{+}$is a finite sum of projections if and only if either $a$ is a projection or $\|a\|>1$.

By employing a suitable notion of essential central spectrum, we proved also that a similar sufficient condition holds also for global properly infinite von Neumann algebras [8, Theorem 4.3].

For multiplier algebras we proved in [9, Theorem 3.3] a similar result: if $\mathcal{A}$ is a $\sigma$-unital, nonunital, purely infinite simple $\mathrm{C}^{*}$-algebra, then every positive element $a \in \mathcal{M}(\mathcal{A}) \backslash \mathcal{A}$ with $\|a\|_{e}>1$ can be expressed as a finite sum of projections.

Since $\sigma$-unital purely infinite simple $\mathrm{C}^{*}$-algebras share some properties of $\sigma$-finite type III factors, one would hope that every positive element $a$ in a purely infinite simple $\mathrm{C}^{*}$-algebra with $\|a\|>1$ could also be written as a finite sum of projections. If not, one would like to see which such algebras do not have this property.

The objective of this paper is to prove the following result.

Theorem 1.1. Let $\mathcal{A}$ be a purely infinite simple $C^{*}$-algebra such that $K_{0}(\mathcal{A})$ is a torsion group; namely, it contains no free elements. Then a positive element $a \in \mathcal{A}^{+}$is a finite sum of projections if and only if either $a$ is a projection or $\|a\|>1$. 
Both in the case of von Neumann algebras ([8]) and in the case of multiplier algebras ([7] and [9]), the results were obtained by first decomposing $a$ into a special infinite sum of projections (converging in the strong topology in the von Neumann algebra case, and in the strict topology in the multiplier algebra case), and then reassembling these sums into finite sums of projections. However, for purely infinite simple $\mathrm{C}^{*}$-algebras the above techniques can no longer be applied, and we will have to use a different approach.

A key reduction is that if a purely infinite simple $\mathrm{C}^{*}$-algebra $\mathcal{A}$ has the property that $\alpha p$ is a finite sum of projections for all $\alpha>1$ and all projections $p \in \mathcal{A}$, then each $a \in \mathcal{A}^{+}$with $\|a\|>1$ is also of a finite sum of projections. The key in this reduction is the fact derived in [9] that each positive element $a \in \mathcal{A}$ can be written as positive linear combination of projections.

Then we prove that in any purely infinite simple $\mathrm{C}^{*}$-algebra, $\alpha p$ is a finite sum of projections for any $\alpha>1$ and any projection $p$ that represents the zero element of $K_{0}(\mathcal{A})$ (Proposition 2.3 ).

This will be done by showing that $\alpha p$ is of a finite sum of projections for any $\alpha \in\left[\frac{3}{2}, 3\right]$ (Lemma 2.1) and then proving that $\alpha p \oplus \beta q$ is a finite sum of projections for any pair of rational numbers $\alpha>1$ and $\beta \geq 0$ and every projection $p \sim q$, which represents the zero element of $K_{0}(\mathcal{A})$ (Lemma 2.2).

Combining these results, we then obtain that $\alpha p \oplus \beta q$ is a finite sum of projections for any $\alpha>1, \beta \geq 0$ and any projections $p, q \in \mathcal{A}$ which represent elements of $K_{0}(\mathcal{A})$ with finite orders (Proposition 2.5).

As a consequence, if all elements of $K_{0}(\mathcal{A})$ are of finite order, i.e., $K_{0}(\mathcal{A})$ is a torsion group, then $a \in \mathcal{A}^{+}$is a finite sum of projections if and only if either $a$ is a projection or $\|a\|>1$ (Theorem 1.1).

Finally, we note that it is an open question whether Theorem 1.1 is true for simple purely infinite $\mathrm{C}^{*}$-algebras with a torsion-free element in the $K_{0}$ group. Indeed, it is open even for the case of the Cuntz algebra $O_{\infty}$.

Question 1.2. Would the conclusion of Theorem 1.1 still be true if we replaced $\mathcal{A}$ with the Cuntz algebra $O_{\infty}$ ?

For the reader's convenience we summarize some basic facts on the $K$-theory of infinite simple $\mathrm{C}^{*}$-algebras that we will use in this paper. Handy references are the original papers by Cuntz [2] and [3].

Recall that $[p]$ denotes the equivalence class of a projection $p \in \mathcal{A}$ for any $\mathrm{C}^{*}$ algebras $\mathcal{A}$. If $\mathcal{A}$ is infinite and simple, then the $K_{0}$ group of $\mathcal{A}$ can be described as follows:

(1) $K_{0}(\mathcal{A})=\{[p] \mid p$ is an infinite projection in $\mathcal{A}\}$.

(2) The addition ' + ' on $K_{0}(\mathcal{A})$ is defined as follows: for any two infinite projections $p, q \in \mathcal{A}$, choose $p^{\prime} \sim p, q^{\prime} \sim q$ so that $p^{\prime} q^{\prime}=0$. Then $[p]+[q]=\left[p^{\prime}+q^{\prime}\right]$. In particular, we denote

$$
n[p]=\overbrace{[p]+[p]+\cdots+[p]}^{n \text { times }} .
$$

(3) The zero (or unit) element $\theta \in K_{0}(\mathcal{A})$ is defined as $\left[p-p^{\prime}\right]$ for any pair of projections $p^{\prime}<p$ with $p^{\prime} \sim p$ and $p-p^{\prime}$ infinite.

(4) For any infinite projection $q \in \mathcal{A}$ and any positive integer $m, q$ can be decomposed into the sum $q=\sum_{j=0}^{m} q_{j}$ of mutually orthogonal projections $q_{j}$, with $q_{0} \sim q$ and $\left[q_{j}\right]=\theta$ for all $j>0$. 
(5) An infinite $\mathrm{C}^{*}$-algebra $\mathcal{A}$ has torsion $K_{0}$ if for every projection $p \neq 0$ the element $[p]$ is finitely cyclic in $K_{0}(\mathcal{A})$; namely, there is a positive integer $n$ for which $n[p]=\theta$, the zero element of $K_{0}(\mathcal{A})$. Among algebras with torsion $K_{0}$ are the Cuntz algebras $\mathcal{O}_{n}$ for $2 \leq n<\infty$, for which $K_{0}\left(\mathcal{O}_{n}\right)=$ $\mathbb{Z} /(n-1) \mathbb{Z}$; in particular, $K_{0}\left(\mathcal{O}_{2}\right)=\{\theta\}$, the trivial group.

\section{Finite sums of ProjeCtions and $K$-TheORY}

This entire section will be devoted to establishing the proof of our main result by a sequence of technical lemmas.

Lemma 2.1. Let $\mathcal{A}$ be an infinite simple $C^{*}$-algebra, let $p \in \mathcal{A}$ be a projection that represents the zero element of $K_{0}(\mathcal{A})$ (i.e., $[p]=\theta$ ) and let $\gamma \in\left[\frac{3}{2}, 3\right]$. Then there are six projections $q_{j} \in \mathcal{A}$, with $q_{j} \sim p$ and such that $\gamma p=\sum_{1}^{6} q_{j}$.

Proof. Decompose $p=p_{1}+p_{2}$ into the sum of two mutually orthogonal projections $p_{1} \sim p_{2} \sim p$. Let

$$
\begin{aligned}
& a_{1}:=(2 \gamma-3) p_{1}+(3-\gamma) p_{2}, \\
& a_{2}:=(3-\gamma) p_{1}+(2 \gamma-3) p_{2} .
\end{aligned}
$$

Then

$$
\gamma p=a_{1}+a_{2} \quad \text { and } \quad a_{1} \geq 0, a_{2} \geq 0 .
$$

Further decompose $p_{2}=p_{2}^{\prime}+p_{2}^{\prime \prime}$ into the sum of two mutually orthogonal projections $p_{2}^{\prime} \sim p_{2}^{\prime \prime} \sim p_{2}$. Then

$$
a_{1}=(2 \gamma-3) p_{1}+(3-\gamma) p_{2}^{\prime}+(3-\gamma) p_{2}^{\prime \prime} .
$$

Since the three projections $p_{1}, p_{2}^{\prime}$, and $p_{2}^{\prime \prime}$ are mutually orthogonal and equivalent, $a_{1}$ belongs to an embedding of $\mathbb{M}_{3}(\mathbb{C})$ in $p \mathcal{A} p$ where $p_{1}, p_{2}^{\prime}$, and $p_{2}^{\prime \prime}$ are identified with rank-one projections. Thus $a_{1}$ can be identified with a positive matrix in $\mathbb{M}_{3}(\mathbb{C})$ with $\operatorname{Tr}\left(a_{1}\right)=3$ and $\operatorname{rank}\left(a_{1}\right) \leq 3$. Then it follows from [5, Theorem 1.1] that $a_{1}$ is the sum of three projections (all equivalent to $p$ ). The same argument applies to $a_{2}$, and hence, the proof is completed.

Lemma 2.2. Let $\mathcal{A}$ be an infinite simple $C^{*}$-algebra, let $p, q \in \mathcal{A}$ be two orthogonal projections that represent the zero element of $K_{0}(\mathcal{A})$, and let $\alpha, \beta$ be rational numbers with $\alpha>1$ and $\beta \geq 0$. Then $a:=\alpha p+\beta q$ is a finite sum of projections.

Proof. Set $\alpha=\frac{k}{m}$ and $\beta=\frac{h}{m}$ with $k, h, m$ integers and choose an integer $r \geq \frac{m}{k-m}$, equivalently, $r k \geq r m+m$. Then decompose $p=\sum_{1}^{r m} p_{j}$ (resp., $q=\sum_{1}^{m} q_{j}$ ) into the sum of $r m$ (resp. $m$ ) mutually orthogonal projections $p_{j} \sim q_{j} \sim p$. Then

$$
a=\frac{k}{m} \sum_{1}^{r m} p_{j}+\frac{h}{m} \sum_{1}^{m} q_{j}
$$

can be identified with a positive matrix in $\mathbb{M}_{r m+m}(\mathbb{C})_{+}$with

$$
\operatorname{Tr}(a)=r k+h \in \mathbb{N} \text { and } \operatorname{rank}(a) \leq r m+m .
$$

Since $r k+h \geq r m+m$, it follows once again from [5, Theorem 1.1] that $a$ is a finite sum of projections. 
Proposition 2.3. Let $\mathcal{A}$ be an infinite simple $C^{*}$-algebra, let $p, q \in \mathcal{A}$ be two orthogonal equivalent projections that represent the zero element of $K_{0}(\mathcal{A})$, and let $\alpha, \beta$ be real numbers with $\alpha>1$ and $\beta \geq 0$. Then $a:=\alpha p+\beta q$ is a finite sum of projections.

Proof. If $\beta=0$, write $\alpha p=\alpha p_{1}+\alpha p_{2}$, where $p_{1} \sim p_{2} \sim p$ and $p_{1} p_{2}=0$. Thus assume without loss of generality that $\beta>0$.

Let $\rho_{1}, \rho_{2}, \rho_{3}$ be rational numbers with

$$
\begin{aligned}
1 & <\rho_{1}<\alpha, \\
\frac{1}{3}\left(\alpha-\rho_{1}\right) & <\rho_{2}<\frac{2}{3}\left(\alpha-\rho_{1}\right), \\
\frac{1}{3} \beta & <\rho_{3}<\frac{2}{3} \beta .
\end{aligned}
$$

Decompose $p=p^{\prime}+p^{\prime \prime}$ into the sum of two mutually orthogonal projections $p^{\prime} \sim$ $p^{\prime \prime} \sim p$. Then

$$
a=\rho_{1} p+\left(\alpha-\rho_{1}\right) p^{\prime \prime}+\left(\alpha-\rho_{1}\right) p^{\prime}+\beta q .
$$

Since $\frac{\alpha-\rho_{1}}{\rho_{2}} \in\left[\frac{3}{2}, 3\right]$ and $\frac{\beta}{\rho_{3}} \in\left[\frac{3}{2}, 3\right]$, by Lemma 2.1 we can find projections $\left\{q_{j}\right\}_{1}^{18}$ with $q_{j} \sim p$ for all $j$ and such that

$$
\begin{aligned}
\frac{\alpha-\rho_{1}}{\rho_{2}} p^{\prime \prime} & =\sum_{1}^{6} q_{j}, \\
\frac{\alpha-\rho_{1}}{\rho_{2}} p^{\prime} & =\sum_{7}^{12} q_{j}, \\
\frac{\beta}{\rho_{3}} q & =\sum_{13}^{18} q_{j} .
\end{aligned}
$$

It follows that

$$
a=\rho_{1} p+\rho_{2} \sum_{1}^{6} q_{j}+\rho_{2} \sum_{7}^{12} q_{j}+\rho_{3} \sum_{13}^{18} q_{j} .
$$

Now decompose $p^{\prime}=\sum_{1}^{6} p_{j}$ and $p^{\prime \prime}=\sum_{7}^{18} p_{j}$ into the sum of mutually orthogonal projections $p_{j} \sim p$. Thus

$$
a=\sum_{1}^{6}\left(\rho_{1} p_{j}+\rho_{2} q_{j}\right)+\sum_{7}^{12}\left(\rho_{1} p_{j}+\rho_{2} q_{j}\right)+\sum_{13}^{18}\left(\rho_{1} p_{j}+\rho_{3} q_{j}\right) .
$$

Since all the coefficients $\rho_{i}$ are rational, $\rho_{1}>1$, and $p_{j} q_{j}=0$ for $1 \leq j \leq 18$ because $p^{\prime} p^{\prime \prime}=p q=0$, it follows by Lemma 2.2 that each of the above 18 summands is a finite sum of projections, and hence, so is $a$.

Taking $\beta=0$ in the above proposition we thus have:

Corollary 2.4. Let $\mathcal{A}$ be an infinite simple $C^{*}$-algebra and let $p$ be a projection in $\mathcal{A}$ that represents the zero element of $K_{0}(\mathcal{A})$. Then for all $\alpha>1$ the positive element $\alpha p$ is a finite sum of projections.

This result extends also to projections that represent finitely cyclic elements of $K_{0}(\mathcal{A})$. 
Proposition 2.5. Let $\mathcal{A}$ be a purely infinite simple $C^{*}$-algebra and let $p$ be a projection of $\mathcal{A}$ that represents a finite order element of $K_{0}(\mathcal{A})$ (i.e., such that $[p]$ is finitely cyclic). Then $\alpha p$ is a finite sum of projections.

Proof. Assume also without loss of generality that $\alpha<2$. Let $\theta$ be the zero element of $K_{0}(\mathcal{A})$. By hypothesis $n[p]=\theta$ for some integer $n \in \mathbb{N}$. Then $\frac{1}{\alpha-1}-\frac{1}{n}>0$ and let $m \geq 0$ be the integer part of $\frac{1}{\alpha-1}-\frac{1}{n}$ and let $0 \leq \delta<1$ be its fractional part, i.e.,

$$
m=\frac{1}{\alpha-1}-\frac{1}{n}-\delta .
$$

Since $[p]=(m+1) \theta+[p]=((m+1) n+1)[p]$, we can decompose $p$ into the sum $p=\sum_{j=0}^{(m+1)(n)} p_{j}$ of mutually orthogonal projection $p_{j} \sim p$. Then

$$
\alpha p=\alpha \sum_{j=0}^{m n} p_{j}+(\alpha-1) \delta \sum_{j=m n+1}^{(m+1) n} p_{j}+(\alpha-(\alpha-1) \delta) \sum_{j=m n+1}^{(m+1) n} p_{j} .
$$

Let

$$
b:=\alpha \sum_{j=0}^{m n} p_{j}+(\alpha-1) \delta \sum_{j=m n+1}^{(m+1) n} p_{j}
$$

and

$$
c:=(\alpha-(\alpha-1) \delta) \sum_{j=m n+1}^{(m+1) n} p_{j}
$$

Then $a=b+c$. Since all the projections $p_{j}$ are mutually orthogonal and equivalent (to $p$ ), the positive operator $b$ can be identified with a finite matrix with $\operatorname{rank}(b) \leq$ $(m+1) n+1$ and with

$$
\operatorname{Tr}(b)=(m n+1) \alpha+n(\alpha-1) \delta=(m+1) n+1 .
$$

Thus, by [5, Theorem 1.1], $b$ is a finite sum of projections. Since $\alpha-(\alpha-1) \delta>1$ and since

$$
\left[\sum_{j=m n+1}^{(m+1) n} p_{j}\right]=n[p]=\theta,
$$

by Corollary 2.4 $c$ too is a finite sum of projections, and hence, so is $a$.

In particular, if $\mathcal{A}$ is a purely infinite simple $\mathrm{C}^{*}$-algebra such that $K_{0}(\mathcal{A})$ is a torsion group, i.e., every element is of finite order, then $\mathcal{A}$ has the property that the element $\alpha p$ is a finite sum of projections for every $\alpha>1$ and every projection $p \in \mathcal{A}$. As we will see, this property extends to all positive operators with norm larger than 1.

Lemma 2.6. Let $\mathcal{A}$ be a purely infinite simple $C^{*}$-algebra with the property that the element $\alpha p$ is a finite sum of projections for every $\alpha>1$ and every projection $p \in \mathcal{A}$. Then for every orthogonal pair of projections $0 \neq p, q \in \mathcal{A}$ and every pair of real numbers $\alpha, \beta$ with $\alpha>1$ and $\beta \geq 0$, the operator $a=\alpha p+\beta q$ is a finite sum of projections.

Proof. Without loss of generality assume that $\beta<1$. Let $n \in \mathbb{N}$ be the integer part of $\frac{2-\beta}{\alpha-1}$, let $0 \leq \delta<1$ be its fractional part and let $\epsilon:=\delta(\alpha-1)$. Then $0 \leq \epsilon<\alpha-1$ and

$$
n \alpha+\beta+\epsilon=n+2 .
$$


Since $[p]=(n+1)[q]+([p]-(n+1)[q])$ in $K_{0}(\mathcal{A})$, we can decompose $p$ into the sum $p=p^{\prime}+\sum_{j=0}^{n} q_{j}$ of mutually orthogonal nonzero projections with $q_{j} \sim q$ for $0 \leq j \leq n$ and a nonzero "remainder" $p^{\prime}$ also orthogonal to all the projections $q_{j}$. Then

$$
a=\alpha p^{\prime}+(\alpha-\epsilon) q_{0}+\epsilon q_{0}+\sum_{1}^{n} \alpha q_{j}+\beta q .
$$

Let $b:=\epsilon q_{0}+\sum_{1}^{n} \alpha q_{j}+\beta q$. Then $b \geq 0$, and since all the projections are mutually orthogonal and equivalent, we identify it with a positive matrix with

$$
\operatorname{Tr}(b)=n \alpha+\beta+\epsilon=n+2 \geq \operatorname{rank}(b) .
$$

Thus $b$ is a finite sum of projections. By hypothesis so are $\alpha p^{\prime}$ and $(\alpha-\epsilon) q_{0}$, and hence, so is $a$.

Theorem 2.7. Let $\mathcal{A}$ be a purely infinite simple $C^{*}$-algebra with the property that the element $\alpha p$ is a finite sum of projections for every $\alpha>1$ and every projection $p \in \mathcal{A}$. Then $a \in \mathcal{A}^{+}$is a finite sum of projections if and only if either $a$ is a projection or $\|a\|>1$.

Proof. If $a$ is a sum of projections, then $\|a\| \geq 1$. Then either the projections are mutually orthogonal, in which case $a$ itself is a projection, or $\|a\|>1$.

For the converse implication, assume that $\|a\|>1$. By passing if necessary to the hereditary algebra $(a \mathcal{A} a)^{-}$generated by $a$ which is $\sigma$-unital, we assume without loss of generality that $\mathcal{A}$ itself is $\sigma$-unital. By [9, Remark 2.1 (II)], $\mathcal{A}$ has the PLP property; namely the positive cone of each of its hereditary $\mathrm{C}^{*}$-subalgebras is the closure of the positive combinations of its projections. But then, by [9] Lemma 2.10], either $a=\alpha p \oplus c$ for some $\alpha>1$ and nonzero projections $p \in \mathcal{A}$ and a positive element $c \in \mathcal{A}$ or $a$ is the sum of two such operators. Assume without loss of generality that $a=\alpha p \oplus c$. By [9, Theorem 2.11], $c=\sum_{1}^{n} \lambda_{j} q_{j}$ for some scalars $\lambda_{j}>0$ and projections $q_{j}$. Decompose $p$ into the sum $p=\sum_{1}^{n} p_{j}$ of mutually orthogonal nonzero projections $p_{j} \in \mathcal{A}$. Then

$$
a=\sum_{1}^{n}\left(\alpha p_{j}+\lambda_{j} q_{j}\right)
$$

where $p_{j} q_{j}=0$ for all $j$. By Lemma 2.6 each summand $\alpha p_{j}+\lambda_{j} q_{j}$ is a finite sum of projections, and hence, so is $a$.

Combining Proposition 2.5 and Theorem 2.7 we thus obtain Theorem 1.1, our main result.

Theorem 1.1, Let $\mathcal{A}$ be a purely infinite simple $C^{*}$-algebra such that $K_{0}(\mathcal{A})$ is a torsion group; namely, it contains no free elements. Then a positive element $a \in \mathcal{A}^{+}$is a finite sum of projections if and only if either $a$ is a projection or $\|a\|>1$.

Remark 2.8. $\quad$ (i) Purely infinite simple $\mathrm{C}^{*}$-algebras with $K_{0}(\mathcal{A})$ a torsion group include of course all those trivial $K_{0}$ and all Cuntz algebras $\mathcal{O}_{n}$ with $2 \leq$ $n<\infty$.

(ii) The proofs of Lemmas 2.1 and 2.2, and Proposition 2.3. and hence, the conclusion in Corollary 2.4 hold also in $\sigma$-finite von Neumann factors if restricted to infinite projections. Namely, if $M$ is a $\sigma$-finite von Neumann factor, then $\alpha p$ is a finite sum of projections for all $\alpha>1$ and all infinite 
projections $p$. This observation provides a simple alternative proof for the fact that $\alpha 1$ is a finite sum of projections in $B(H)$ if $\alpha>1$ (see [10], [11, [13, [1, 8]).

(iii) Notice explicitly that the techniques of the present paper do not provide an alternative proof for the sufficiency of the condition $\|a\|_{e}>1$ for $a \in B(H)^{+}$ to be a finite sum of projections. Indeed, while by a proof similar to the one for Theorem 1.1 (see 8, Theorem 4.3]) it is enough to consider elements of the form $a=\alpha p \oplus \beta q$, where $\alpha>1, \beta \geq 0, p, q$ are projections, and $p$ is infinite, it is not enough to consider only elements where $q$ also is infinite. But the proofs of Proposition 2.3 or of Lemma 2.6 do not apply unless $q$ too is infinite; a different technique is required when $q$ is finite (see 8 , Lemma 4.2]).

Notice also that the condition $\|a\|_{e}>1$ implies that $\pi(a)$ is a finite sum of projections in the Calkin algebra $B(H) / K(H)$, but this alone does not imply that the same holds for $a$ (e.g., there are cases of operators $1+k$ with $k \in K(H)^{+}$that are not finite sum of projections (see [8, Example 5.9]).

(iv) Technical difficulties occur at attempting the analysis of purely infinite simple $\mathrm{C}^{*}$-algebras whose $K_{0}$ group contains free elements; in particular, even $\mathcal{O}_{\infty}$ resists all efforts so far. Unlike all type III von Neumann factors in which all hereditary $\mathrm{W}^{*}$-subalgebras are unital, hereditary $\mathrm{C}^{*}$-subalgebras of a $\sigma$-unital purely infinite simple $\mathrm{C}^{*}$-algebra $\mathcal{A}$ are either unital or stable (namely, $\mathcal{A} \cong \mathcal{A} \otimes K(H)$ ), where $\mathcal{A}$ can be chosen unital and $K(H)$ is the stable algebra of all compact operators on a separable Hilbert space (see [14] and [15]). It is well known that infinite rank positive operators in $K(H)$ are not finite sums of projections, but the same positive elements in the canonical embedding of $K(H)$ as a $\mathrm{C}^{*}$-subalgebra of $\mathcal{A} \otimes K(H)$ become finite sums of projections as long as $K_{0}(\mathcal{A})$ is a torsion group. Whether the same conclusion can be expected or not when $K_{0}(\mathcal{A})$ is not a torsion group remains mysterious. If not, these purely infinite simple $\mathrm{C}^{*}$-algebras would exhibit quite a different behavior from type III factors concerning the decompositions of its positive elements.

\section{REFERENCES}

[1] Choi, M.D. and Wu, P.Y., Sums of orthogonal projections. Preprint.

[2] Cuntz, J., Simple $C^{*}$-algebras generated by isometries, Comm. Math. Phys., 57 (1977), 173185. MR0467330 (57:7189)

[3] Cuntz, J., K-theory for certain $C^{*}$-algebras, Ann. of Math. (2), 131 (1981), 181-197. MR604046 (84c:46058)

[4] Dykema, K., Freeman, D., Kornelson, K., Larson, D., Ordower, M., and Weber, E., Ellipsoidal tight frames and projection decompositions of operators. Illinois J. of Math. 48 (2004), 477489. MR2085421 (2005e:42092)

[5] Fillmore, P., On sums of projections. J. Func. Anal. 4 (1969), 146-152. MR0246150 (39:7455)

[6] Kaftal, V., Ng, P. W., and Zhang, S., Strong sums of projections in von Neumann factors. J. Funct. Anal. 257 (2009), 2497-2529. MR2555011(2011b:46096)

[7] Kaftal, V., Ng, P. W., and Zhang, S., Projection decomposition in multiplier algebras. Math. Ann., to appear.

[8] Kaftal, V., Ng, P. W., and Zhang, S., Finite sums of projections in von Neumann algebras 2010. Trans. Amer. Math. Soc., to appear.

[9] Kaftal, V., Ng, P. W., and Zhang, S., Positive combinations and sums of projections in purely infinite simple $C^{*}$-algebras and their multiplier algebras. Proc. Amer. Math. Soc. 139 (2011), no. 8, 2735-2746. MR2801613 
[10] Kruglyak, S., Rabanovich, V., and Samoŭlenko, Y., On sums of projections. Funct. Anal. and Appl., 36 (2002), 182-195. MR.1935900 (2004e:47021)

[11] Kruglyak, S., Rabanovich, V., and Samoulenko, Y., Decomposition of a scalar matrix into a sum of orthogonal projections. Lin. Alg. and Applic., 370 (2003), 217-225. MR1994329 (2004f:15045)

[12] Marcoux, L., Projections, commutators and Lie ideals in $C^{*}$-algebras, Mathematical Proceedings of the Royal Irish Academy, 110A (2010), no. 1, 31-55. MR2666670

[13] Wu, P.Y., Additive combinations of special operators. Funct. Anal. Oper. Theory., Banach Ctr. Publ., Inst. Math. Polish Acad. Sci., 30 (1994), 337-361. MR.1285620 (95d:47018)

[14] Zhang, S., A property of purely infinite simple $C^{*}$-algebras. Proc. Amer. Math. Soc., 109 (1990) no. 3, 717-720. MR1010004 (90k:46134)

[15] Zhang, S., Certain $C^{*}$-algebras with real rank zero and their corona and multiplier algebras. II, $K$-Theory 6 (1992), no. 1, 1-27. MR1186771 (94i:46094)

Department of Mathematics, University of Cincinnati, P. O. Box 210025, Cincinnati, Оніо 45221-0025

E-mail address: kaftalv@ucmail.uc.edu

Department of Mathematics, University of Louisiana, 217 Maxim D. Doucet Hall, P.O. Box 41010, Lafayette, Louisiana 70504-1010

E-mail address: png@louisiana.edu

Department of Mathematics, University of Cincinnati, P.O. Box 210025, Cincinnati, Оніо 45221-0025

E-mail address: zhangs@math.uc.edu 